\title{
HETEROCLINIC ORBITS AND CHAOTIC DYNAMICS IN PLANAR FLUID FLOWS*
}

\author{
ANDREA LOUISE BERTOZZI $\dagger$
}

\begin{abstract}
An extension of the planar Smale-Birkhoff homoclinic theorem to the case of a heteroclinic saddle connection containing a finite number of fixed points is presented. This extension is used to find chaotic dynamics present in certain time-periodic perturbations of planar fluid models. Specifically, the Kelvin-Stuart cat's eye flow is studied, a model for a vortex pattern found in shear layers. A flow on the two-torus with Hamiltonian $H_{0}=(2 \pi)^{-1} \sin \left(2 \pi x_{1}\right) \cos \left(2 \pi x_{2}\right)$ is studied, as well as the evolution equations for an elliptical vortex in a three-dimensional strain flow.
\end{abstract}

Key words. homoclinic orbits, Melnikov's method, Kelvin-Stuart cat's eyes, elliptical vortices

AMS(MOS) subject classifications. 34C35, 54H20, 58F08, 58F13, 70K99, 76C05

1. Introduction. Organized vortex structures in two-dimensional fluid flows can often be viewed as planar dynamical systems with multiple heteroclinic saddle connections. We wish to study how such saddle connections break up under small perturbations. In the homoclinic case, the Smale-Birkhoff Theorem and Melnikov's method are two useful tools for studying the onset of chaos and mixing in planar flows possessing a simple homoclinic orbit. We extend the planar homoclinic theorem to the case of a heteroclinic orbit connecting a finite number of saddle points, enabling us to analyze fluid models to which the original homoclinic theory does not apply.

We present three planar fluid models that exhibit heteroclinic saddle connections. The Kelvin-Stuart cat's eye flow is a well-known model for a pattern found in shear layers. This flow is a planar dynamical system possessing an infinite number of heteroclinic saddle connections involving two fixed points each. We also study a planar lattice flow in which we find groups of four saddle points linked by heteroclinic orbits. The lattice flow is an interesting model for certain convection patterns as well as for nonlinear Taylor vortex flow. In the unperturbed case, these flows are steady solutions to the inviscid Euler equations and thus have a direct Hamiltonian formulation. We apply the simplified Hamiltonian form of Melnikov's method to find chaotic motion and mixing occurring in time-periodic perturbations of these two planar flows.

The third application of Melnikov's method presented here is of a somewhat different nature from the first two. We examine the evolution equations for an elliptical vortex in an imposed strain. These equations have a Hamiltonian form based on a dimensionless time parameter. The most physically interesting perturbations are based on real time and so we are forced to study a non-Hamiltonian dynamical system with a homoclinic orbit. We apply the non-Hamiltonian version of Melnikov's method to find chaotic dynamics occurring in the case of periodic stretching of the straining flow in a third dimension.

2. Extension of the homoclinic theorem and Melnikov's method. The ideas for the homoclinic theorem were first laid out by Birkhoff [5] and were developed by Smale [26]. We consider a planar diffeomorphism $\varphi$ possessing a hyperbolic saddle point $p$ whose stable and unstable manifolds intersect transversely at a point $q$. A result of this theorem is that $\varphi$ possesses a subsystem equivalent to a shift on two symbols. We extend this theorem to the case of $N$ fixed points joined by transverse saddle connections

\footnotetext{
* Received by the editors October 1, 1987; accepted for publication January 21, 1988.

$\dagger$ Department of Mathematics, Princeton University, Princeton, New Jersey 08544.
} 
(see Fig. 2.3 for the case $N=3$ ). The homoclinic theorem is proved by constructing the horseshoe map and showing that it possesses the shift as a subsystem (Moser [19]). We must then show that $\varphi$ possesses the horseshoe map as a subsystem. Keeping in mind Moser's proof of the homoclinic theorem, we construct the generalized horseshoe map, and present a sketch of the heteroclinic theorem. For the complete details the reader is referred to [4].

2.1. The horseshoe map and the shift on two symbols. We first define the horseshoe map used in the homoclinic case. The horseshoe map is a topological mapping of the unit square $Q$ into the plane such that $\varphi(Q) \cap Q$ has two components $U_{1}$ and $U_{2}$. The pre-images of $U_{1}$ and $U_{2}$ are denoted by $V_{i}=\varphi^{-1}\left(U_{i}\right), i=1,2 . V_{1}$ and $V_{2}$ are vertical strips connecting the upper and lower edges of $Q$ (see Fig. 2.1). The iterates $\varphi^{k}$ of $\varphi$ are not defined in all of $Q$, so we construct the invariant set

$$
I=\bigcap_{k=-\infty}^{\infty} \varphi^{-k}(Q)
$$

in which all iterates $\varphi^{k}$ are defined. Associated with each point $p$ of $I$ is a bi-infinite sequence $\left(\cdots s_{-1}, s_{0} ; s_{1}, s_{2} \cdots\right), s_{i} \in\{1,2\}$ of ones and twos, where $\varphi^{-k}(p) \in V_{s_{k}}$ or

$$
p \in \bigcap_{k=-\infty}^{\infty} \varphi^{k}\left(V_{s_{k}}\right)
$$

On the set $S$ of all such sequences, we define a map $\sigma$ by $(\sigma s)_{i}=s_{i+1}$. Under the map $\sigma$, all the elements of $s$ are shifted over by one. This provides a mapping $\tau: I \rightarrow S$ with $\left.\tau \varphi\right|_{I}=\sigma \tau$ as long as $\tau$ is invertible. We introduce a topology on $S$ as follows: Given $s^{*}=\left(\cdots, s_{-2}^{*}, s_{-1}^{*}, s_{0}^{*} ; s_{1}^{*}, s_{2}^{*}, \cdots\right) \in S$ then $U_{j}=\left\{s \in S \mid s_{k}=s_{k}^{*},(|k|<j)\right\}$ form a neighborhood basis for $s^{*}$. We see that the horseshoe map possesses periodic orbits of arbitrary period, as well as an orbit that comes arbitrarily close to all points of $I$. This last orbit is obtained by constructing a sequence that contains all possible finite strings of ones and twos.

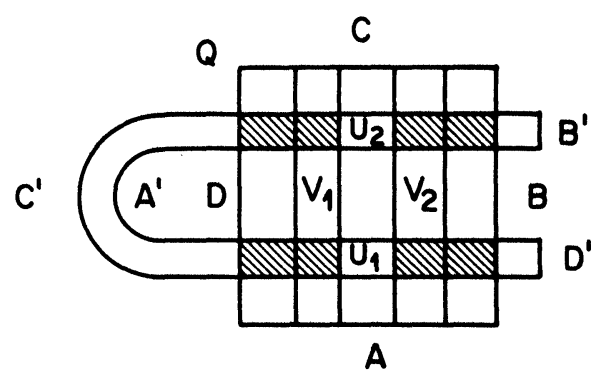

FIG. 2.1. The horseshoe map.

2.2. A generalization of the horseshoe map. Consider a set of $N$ disjoint squares $Q_{i}$ in the plane and a map $\varphi: \cup Q_{i} \rightarrow \mathbb{R}^{2}$ such that $\varphi\left(Q_{i}\right) \cap Q_{i}$ is a horizontal strip in $Q_{i}$ and $\varphi\left(Q_{i}\right) \cap Q_{i+1(\bmod N)}$ is a horizontal strip in $Q_{i+1(\bmod N)}$. Here it is not important how each square $Q_{i}$ is oriented with respect to the other squares, only that $\varphi\left(\cup_{i} Q_{i}\right) \cap Q_{j}$ are horizontal strips in $Q_{j}$ (see Fig. 2.2). Our invariant set thus will be

$$
I=\bigcap_{k=-\infty}^{\infty} \varphi^{-k}\left(\bigcup_{i} Q_{i}\right)
$$




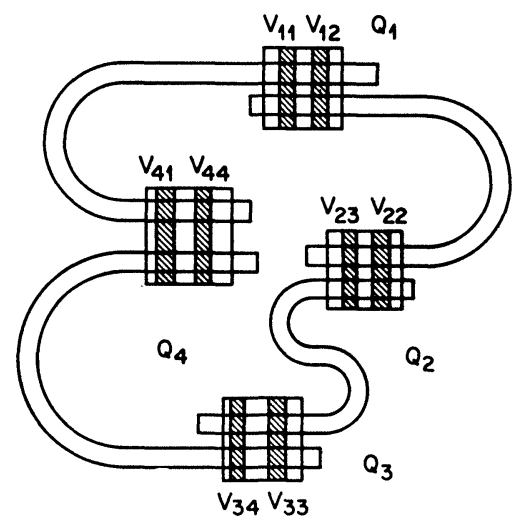

FIG. 2.2

We will associate with each point $p \in I$ a bi-infinite sequence $\left(\cdots, s_{-1}, s_{0} ; s_{1}, s_{2} \cdots\right) \in$ $S^{\prime}$ of $N$ consecutive symbols where

$$
S^{\prime}=\left\{s \mid s_{i} \in\{1, \cdots, N\}, \quad s_{i+1}=s_{i} \quad \text { or } \quad s_{i+1}=s_{i}+1 \quad(\bmod N)\right\}
$$

such that $\varphi^{-k}(p) \in Q_{s_{k}}$. Under the appropriate conditions there is a one-to-one correspondence between points of $I$ and sequences $s \in S^{\prime}$. For the precise details of the above construction as well as a proof of the fact that $I$ and $S^{\prime}$ are topologically isomorphic, the reader is referred to [4].

\subsection{A heteroclinic theorem.}

THEOREM 2.3.1. If a diffeomorphism $\varphi: \mathbb{R}^{2} \rightarrow \mathbb{R}^{2}$ possesses $N$ fixed points $p_{1}, p_{2}, \cdots, p_{N}$ that are nondegenerate hyperbolic saddle points, and there exist points $q_{i}$ at which the unstable manifold $W^{u}\left(p_{i}\right)$ intersects the stable manifold $W^{s}\left(p_{i+1(\bmod N)}\right)$ transversely for all $i$, then $\varphi$ possesses an invariant set I on which some iteration $\varphi^{k}$ is homeomorphic to the shift on $S^{\prime}$, the set of bi-infinite sequences of $N$ consecutive symbols (as described in the preceding section).

We provide an outline of the proof. For details, the reader is referred to [4]. We want to show that $\varphi$ possesses a subsystem satisfying the requirements for the generalized horseshoe map of $\S 2.2$. The stable and unstable manifolds are depicted in Fig. 2.3 (for the case $N=3$ ).

Claim. We can choose an integer $k$ and neighborhoods $U_{i}$ of $p_{i}$ such that the following conditions are satisfied (see Fig. 2.4):

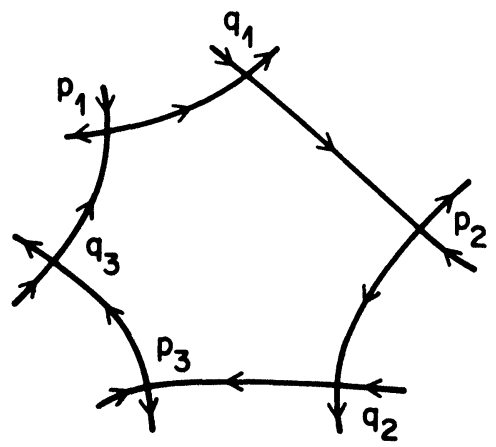

FIG. 2.3 


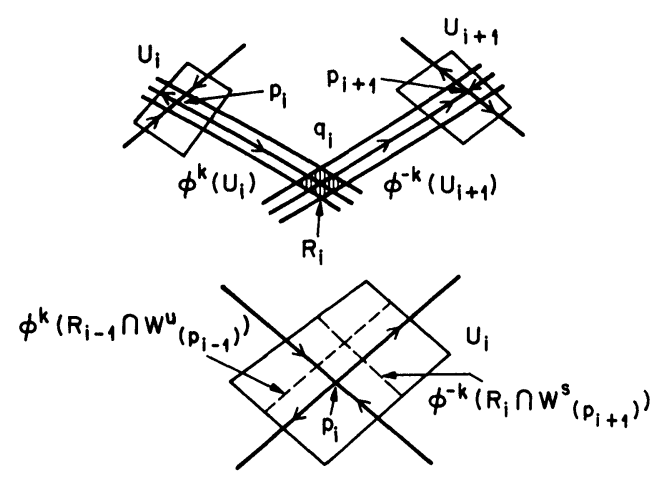

FIG. 2.4

(1) There exists a local coordinate system in $U_{i}$ so that $\varphi$ is linear, and $U_{i}$ is the unit square.

(2) $q_{i} \in \varphi^{k}\left(U_{i}\right)$ and $q_{i} \in \varphi^{-k}\left(U_{i+1(\bmod N)}\right)$ for all $i$.

(3) For $R_{i}=\varphi^{k}\left(U_{i}\right) \cap \varphi^{-k}\left(U_{i+1(\bmod N)}\right)$, we have $\varphi^{-k}\left(R_{i} \cap W^{s}\left(p_{i+1(\bmod N)}\right)\right)$ intersects $\varphi^{k}\left(R_{i-1(\bmod N)} \cap W^{u}\left(p_{i-1(\bmod N)}\right)\right)$ transversely in exactly one point.

We choose $U_{i}$ so that (1) is satisfied for all $i$. Note that if we shrink each $U_{i}$, (1) will still hold. Given any $U_{i}$ satisfying (1), by the definition of stable and unstable manifolds, there exists a $k$ such that (2) is satisfied. Note that $k$ depends on the sizes of the $U_{i}$, which we will continue to shrink until all the above conditions are satisfied. By the $\lambda$-lemma of Palis [23], $\varphi^{-k}\left(R_{i} \cap W^{s}\left(p_{i+1(\bmod N)}\right)\right)$ approaches $W^{s}\left(p_{i}\right)$ and $\varphi^{k}\left(R_{i-1(\bmod N)} \cap W^{u}\left(p_{i-1(\bmod N)}\right)\right)$ approaches $W^{u}\left(p_{i}\right)$ as $k \rightarrow \infty$. Thus for $k$ sufficiently large and the $U_{i}$ sufficiently small, (3) is satisfied. Transversal intersection results because $W^{s}\left(p_{i}\right)$ and $W^{u}\left(p_{i}\right)$ intersect transversely at $p_{i}$. Once (3) is achieved, we can find $U_{i}$ sufficiently small so that $\varphi^{-k}\left(R_{i}\right)$ is a vertical strip and $\varphi^{k}\left(R_{i-1(\bmod N)}\right)$ is a horizontal strip in $U_{i}$. Thus, $\varphi^{2 k}$ possess a subsystem equivalent to the generalized horseshoe map, which in turn possesses a subsystem topologically equivalent to the shift on $N$ consecutive symbols.

This last subsystem is termed "chaotic" because of the interesting properties it exhibits under iterations of $\varphi^{2 k}$. We have orbits of arbitrary period greater than $N$ as well as dense orbits. The bi-infinite sequence corresponding to a dense orbit is formed by concatenating all possible finite sequences of consecutive symbols. We further note the unpredictability of this subsystem. Any two orbits with sequences that agree for some finite length may have completely different sequences further on. Physically we will find these orbits near each other under a finite number of iterations of $\varphi^{2 k}$, yet the orbits diverge as we proceed past the point where their sequences agree. Thus, knowing where a point will be for a fixed finite time in no way predicts where it will be at later times.

2.4. Melnikov's method. Melnikov [18] devised a method for finding the transverse intersection of stable and unstable manifolds given a time-periodic perturbation of a system with a saddle connection. We present the theorem without proof.

Consider the following planar dynamical system:

$$
\dot{x}=f(x)+\varepsilon g(x, t), \quad x \in \mathbb{R}^{2}, \quad g(x, t)=g(x, t+T), \quad 0 \leqq \varepsilon \ll 1,
$$

where for $\varepsilon=0$ we have a saddle connection $\Gamma_{0}$ between two nondegenerate hyperbolic saddle points $p_{1}$ and $p_{2}$ (see Fig. 2.5). The unstable manifold $W_{0}^{u}\left(p_{1}\right)$ of $p_{1}$ and the 


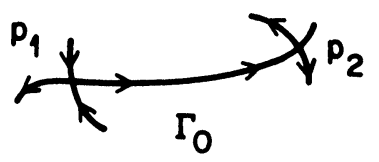

FIG. 2.5

stable manifold $W_{0}^{s}\left(p_{2}\right)$ of $p_{2}$ coincide. Here we include the homoclinic case where $p_{1}=p_{2}$. Associated with $(\mathrm{A})$ is the suspended system

$$
\dot{x}=f(x)+\varepsilon g(x, \theta), \quad(x, \theta) \in \mathbb{R}^{2} \times S^{1} \quad\left(S^{1}=\mathbb{R} / T\right) .
$$

For $\varepsilon$ sufficiently small, (B) possesses a Poincaré map: $P_{\varepsilon}^{t_{0}}: \Sigma_{t_{0}} \rightarrow \Sigma_{t_{0}}$ where $\Sigma_{t_{0}}=$ $\left\{(x, \theta) \in \mathbb{R}^{2} \times S^{1} \mid \theta=t_{0}\right\}$ is a global cross-section of the flow. Let $F_{t}^{\varepsilon}\left(x_{0}, t_{0}\right)$ be the flow map of (B) on $\mathbb{R}^{2} \times S^{1}$. $P_{\varepsilon}^{t_{0}}$ is obtained by a projection onto the first factor: $P_{\varepsilon}^{t_{0}}(x)=$ $\pi\left(F_{T}^{\varepsilon}\left(x, t_{0}\right)\right)$ where $\pi((x, \theta))=x$. Here $P_{\varepsilon}^{t_{0}}$ is a map from $\mathbb{R}^{2}$ to $\mathbb{R}^{2}$.

Our assumptions imply that for $\varepsilon=0, P_{\varepsilon}^{t_{0}}(x)$ has fixed points at $p_{1}$ and $p_{2}$ and so the suspended system has circular orbits $\gamma^{1}=p_{1} \times S^{1}, \gamma^{2}=p_{2} \times S^{1}$ with stable and unstable manifolds $W_{0}^{u}\left(\gamma^{1}\right)$ and $W_{0}^{s}\left(\gamma^{2}\right)$ coinciding to form a "cylinder" $\Gamma_{0} \times S^{1}$. Such saddle connections are quite unstable and thus are expected to break under small perturbations.

We define the Melnikov function

$$
M\left(t_{0}\right)=\int_{-\infty}^{\infty} d\left(q^{0}\left(t-t_{0}\right)\right) \wedge g\left(q^{0}\left(t-t_{0}\right), t\right) \exp \left(\int_{0}^{t-t_{0}} \operatorname{tr} D f\left(q^{0}(s)\right) d s\right) d t,
$$

where $q^{0}(t)$ is the solution to the unperturbed equation (A) starting at $t_{0}$ on the saddle connection $\Gamma_{0}$. We define the wedge product by $a \wedge b=a_{1} b_{2}-b_{1} a_{2}$.

In the case where the unperturbed system is Hamiltonian, we have $\operatorname{tr} D f\left(q^{0}\right)=0$ and the Melnikov function becomes

$$
M\left(t_{0}\right)=\int_{-\infty}^{\infty} f\left(q^{0}\left(t-t_{0}\right)\right) \wedge g\left(q^{0}\left(t-t_{0}\right), t\right) d t .
$$

The examples of $\S \S 3$ and 4 are both Hamiltonian systems. Two useful forms for computation are

$$
M\left(t_{0}\right)=\int_{-\infty}^{\infty} f\left(q^{0}(t)\right) \wedge g\left(q^{0}(t), t+t_{0}\right) \exp \left(\int_{0}^{t} \operatorname{tr} D f\left(q^{0}(s)\right) d s\right) d t
$$

in the non-Hamiltonian case and

$$
M\left(t_{0}\right)=\int_{-\infty}^{\infty} f\left(q^{0}(t)\right) \wedge g\left(q^{0}(t), t+t_{0}\right) d t
$$

in the Hamiltonian case. We note that $M\left(t_{0}\right)$ is itself a periodic function in $t_{0}$. Using the second form, we have that

$$
\begin{aligned}
M\left(t_{0}+T\right) & =\int_{-\infty}^{\infty} f\left(q^{0}(t)\right) \wedge g\left(q^{0}(t), t+t_{0}+T\right) \exp \left(\int_{0}^{t} \operatorname{tr} D f\left(q^{0}(s)\right) d s\right) d t \\
& =\int_{-\infty}^{\infty} f\left(q^{0}(t)\right) \wedge g\left(q^{0}(t), t+t_{0}\right) \exp \left(\int_{0}^{t} \operatorname{tr} D f\left(q^{0}(s)\right) d s\right) d t \\
& =M\left(t_{0}\right),
\end{aligned}
$$

since $g(x, t+T)=g(x, t)$. 
Melnikov's TheOREM. Given the above conditions, and $\varepsilon$ sufficiently small, if $M\left(t_{0}\right)$ has simple zeros, then $W_{\varepsilon}^{s}\left(p_{\varepsilon}^{2}\right)$ and $W_{\varepsilon}^{u}\left(p_{\varepsilon}^{1}\right)$ intersect transversely. If $M\left(t_{0}\right)$ has no zeros in $t_{0} \in[0, T]$ then $W_{\varepsilon}^{s}\left(p_{\varepsilon}^{2}\right) \cap W_{\varepsilon}^{u}\left(p_{\varepsilon}^{1}\right)=\varnothing$.

For a concise proof of the homoclinic case, the reader is directed to Guckenheimer and Holmes [8]. The heteroclinic proof is an obvious generalization. For details, the reader is referred to [4].

3. Kelvin-Stuart cat's eye flow. Consider the following flow in the plane:

$$
\begin{aligned}
& \dot{x}=\frac{a \sinh y}{a \cosh y+\sqrt{a^{2}-1} \cos x}, \\
& \dot{y}=\frac{\sqrt{a^{2}-1} \sin x}{a \cosh y+\sqrt{a^{2}-1} \cos x} .
\end{aligned}
$$

This is a Hamiltonian system with $H_{0}=\log \left(a \cosh y+\sqrt{a^{2}-1} \cos x\right)$. It is a model for a pattern found in shear layer flow (see [27], [12]). The parameter $a$ controls the shape of the cat's eye with a larger $a$ corresponding to wider "eyes." Here we consider only $a>1$. Streamlines are constants of $H_{0}$ (see Fig. 3.1).

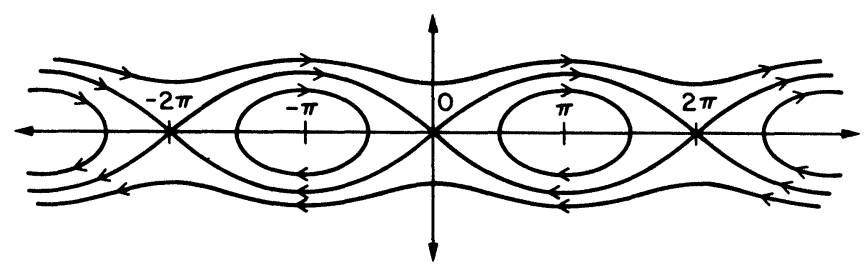

FIG. 3.1

We have fixed points at $(2 \pi N, 0)$ that satisfy the conditions for Melnikov's method. Consider the upper trajectory $\left(x_{0}(t), y_{0}(t)\right)$ from $(0,0)$ to $(2 \pi, 0)$. Along this trajectory we have $x_{0}$ satisfying the equation

$$
\dot{x}_{0}=a \sqrt{\left(\frac{a}{\sqrt{a^{2}-1}}+1-\cos x_{0}\right)^{2}\left(\frac{a^{2}-1}{a^{2}}\right)-1} /\left(a+\sqrt{a^{2}-1}\right) .
$$

This implicitly defines $x_{0}$ by

$$
t=\int_{\pi}^{x_{0}}\left(a+\sqrt{a^{2}-1}\right) d x / a \sqrt{\left(\frac{a^{2}-1}{a^{2}}\right)\left(\cos x-\frac{a}{\sqrt{a^{2}-1}}-1\right)^{2}-1} .
$$

By changing variables to $s=1-\cos x$, this integral becomes

$$
\int_{2}^{1-\cos x_{0}}\left(\left(\frac{a}{\sqrt{a^{2}-1}}+1\right) / s \sqrt{\left(s+\frac{2 a}{\sqrt{a^{2}-1}}\right)(2-s)}\right) d s .
$$

This can be solved exactly to yield

$$
\begin{gathered}
\cos x_{0}=1-\left(\frac{8 a}{a+\sqrt{a^{2}-1}}\right)\left(\frac{1}{e^{\gamma t}+\beta+e^{-\gamma t}}\right), \\
\gamma=\left(\frac{\sqrt{a^{2}-1}}{a+\sqrt{a^{2}-1}}\right) \sqrt{\frac{4 a}{\sqrt{a^{2}-1}}}, \quad \beta=2 \frac{a-\sqrt{a^{2}-1}}{a+\sqrt{a^{2}-1}}
\end{gathered}
$$

along the upper saddle connection from $(0,0)$ to $(2 \pi, 0)$. 
3.1. Periodic stretching of the cat's eye flow. Instead of examining a general perturbation $g(\vec{x}, t)$, consider a perturbation of the parameter $a$. If we take $a$ to be a time-varying parameter of the form $a_{0}+\varepsilon b(t)$, where $b(t)$ is periodic with period $T$, we get a phase diagram where the "cat's eyes" are periodically stretched and compressed by an $\varepsilon$ amount. This corresponds to a time-dependent solution to the Euler equation with external force.

To first order in $\varepsilon$, our perturbed equation is

$$
\begin{aligned}
& \dot{x}=\frac{a_{0} \sinh y}{a_{0} \cosh y+\sqrt{a_{0}^{2}-1} \cos x}-\frac{\varepsilon b(t) \sinh y \cos x}{\sqrt{a_{0}^{2}-1}\left(a_{0} \cosh y+\sqrt{a_{0}^{2}-1} \cos x\right)^{2}}, \\
& \dot{y}=\frac{\sqrt{a_{0}^{2}-1} \sin x}{a_{0} \cosh y+\sqrt{a_{0}^{2}-1} \cos x}+\frac{\varepsilon b(t) \sin x \cosh y}{\sqrt{a_{0}^{2}-1}\left(a_{0} \cosh y+\sqrt{a_{0}^{2}-1} \cos x\right)^{2}} .
\end{aligned}
$$

Thus the driving force for our perturbation is

$$
\vec{F}_{\varepsilon}=\frac{\varepsilon b^{\prime}(t)}{\sqrt{a_{0}^{2}-1}} e^{-2 H_{0}(x, y)}\left(\begin{array}{c}
-\sinh y \cos x \\
\sin x \cosh y
\end{array}\right) .
$$

The perturbed Hamiltonian for this system is

$$
\begin{aligned}
H & =H_{0}+\frac{\varepsilon b(t)}{\sqrt{a_{0}^{2}-1}}\left(\frac{\sqrt{a_{0}^{2}-1} \cosh y+a_{0} \cos x}{a_{0} \cosh y+\sqrt{a_{0}^{2}-1} \cos x}\right) \\
& =H_{0}+H_{1} .
\end{aligned}
$$

Along all streamlines of the unperturbed flow,

$$
H_{1} \propto b(t)\left(\sqrt{a_{0}^{2}-1} \cosh y+a_{0} \cos x\right) .
$$

Since the saddle connections are streamlines of the unperturbed flow, how they break up under a perturbation depends only on the perturbation at the points of the saddle connection. Thus, the Melnikov function for the above perturbation is identical to the one corresponding to the simpler perturbation

$$
H_{1}=\varepsilon b(t)\left(\sqrt{a^{2}-1} \cosh y+a \cos x\right) .
$$

If we let $b(t)$ have the form $\cos (k t)$, then this perturbation corresponds to the superposition of four waves:

$$
\sqrt{a^{2}-1} \cosh y\left(e^{i(z-k t)}+e^{i(z+k t)}\right)+a\left(e^{i(x-k t)}+e^{i(x+k t)}\right) .
$$

Here $z$ is the third coordinate and we take the cross-sectional flow in the plane $z=0$. The wavelength of the perturbation is exactly equal to the length of one of the cat's eyes. The wave speed is allowed to vary.

3.2. The Melnikov function for periodic stretching. Consider the upper trajectory $\left(x_{0}(t), y_{0}(t)\right)$ from $(0,0)$ to $(2 \pi, 0)$ for the unperturbed system.

The Melnikov function for this trajectory is

$$
\begin{aligned}
M\left(t_{0}\right)=\int_{-\infty}^{\infty} C_{1}\left[\left(a_{0} \sin x_{0}(t) \cosh y_{0}(t) \sinh y_{0}(t)\right.\right. \\
\\
\left.\left.\quad+\sqrt{a_{0}^{2}-1} \sinh y_{0}(t) \cos x_{0}(t) \sin x_{0}(t) b\left(t+t_{0}\right)\right)\right] d t,
\end{aligned}
$$

which can be reduced to

$$
M\left(t_{0}\right)=\int_{-\infty}^{\infty} C_{2}\left(\sin x_{0}(t) \sinh y_{0}(t) b\left(t+t_{0}\right)\right) d t
$$


where

$$
C_{2}=\frac{1}{\sqrt{a_{0}^{2}-1}\left(a_{0}+\sqrt{a_{0}^{2}-1}\right)^{2}} .
$$

Here we have exploited the fact that

$$
a_{o} \cosh y_{0}(t)+\sqrt{a_{0}^{2}-1} \cos x_{0}(t)=a_{0}+\sqrt{a_{0}^{2}-1}
$$

We expand $b(t)$ into its Fourier series:

$$
b(t)=\sum_{-\infty}^{\infty}\left(a_{k} \sin k t+b_{k} \cos k t\right) .
$$

The above Melnikov integral then becomes

$$
\begin{aligned}
& \sum_{k=-\infty}^{\infty} \int_{-\infty}^{\infty} C_{2} \sin x_{0}(t) \sinh y_{0}(t)\left(a_{k} \sin k\left(t+t_{0}\right)+b_{k} \cos k\left(t+t_{0}\right)\right) d t \\
& =\sum_{k=-\infty}^{\infty}\left(\left(a_{k} \cos k t_{0}-b_{k} \sin k t_{0}\right) \int_{-\infty}^{\infty} C_{2} \sin x_{0}(t) \sinh y_{0}(t) \sin (k t) d t\right) \\
& =\sum_{k=-\infty}^{\infty}\left(\left(a_{k} \cos \left(k t_{0}\right)-b_{k} \sin \left(k t_{0}\right)\right) M_{0}(k)\right),
\end{aligned}
$$

where we define

$$
\begin{aligned}
M_{0}(k) & =\int_{-\infty}^{\infty} C_{3} \frac{d}{d t}\left[\cos x_{0}(t)\right] \sin (k t) d t, \\
C_{3} & =-\left(a_{0}+\sqrt{a_{0}^{2}-1}\right) C_{2} .
\end{aligned}
$$

We have used the fact that $\sin x_{0}(t) \sinh y_{0}(t)$ is an odd function in $t$. Thus,

$$
\begin{gathered}
M_{0}(k)=\int_{-\infty}^{\infty} C_{4} \frac{e^{\gamma t}-e^{-\gamma t}}{\left(e^{\gamma t}+\beta_{0}+e^{-\gamma t}\right)^{2}} \sin (k t) d t, \\
C_{4}=C_{3}\left(\gamma\left(\frac{8 a_{0}}{a_{0}+\sqrt{a_{0}^{2}-1}}\right)\right) .
\end{gathered}
$$

Evaluation by residues (see Appendix A) yields, for $k \neq 0$,

$$
\begin{gathered}
\left(\frac{\gamma}{2 \pi C_{4}}\right) M_{0}(k)=\left[\frac{m e^{-|m| \alpha}}{2 \sin \alpha}-\left(\frac{e^{-|m| 2 \pi}}{1-e^{-|m| 2 \pi}}\right)\left(\frac{m \sinh |m| \alpha}{\sin \alpha}\right)\right], \\
m=\frac{k}{\gamma}, \quad \alpha=\cos ^{-1}\left(\frac{\beta_{0}}{2}\right), \quad 0<\alpha<\pi / 2 .
\end{gathered}
$$

Whether or not $M\left(t_{0}\right)$ has simple zeros depends on the values of $a_{k}$ and $b_{k}$. For instance, if $b(t)$ is of the form cos $k t$, then we see that $M\left(t_{0}\right)$ has simple zeros for almost all $k$. A similar analysis shows that the lower trajectory has a Melnikov function that is just the negative of the one for the upper trajectory. Since both trajectories break up under the same perturbation to yield the transverse intersection of stable and unstable manifolds, we have satisfied the requirements for the heteroclinic theorem (Theorem 2.3.1) with $N=2$. Our perturbed system has a chaotic subsystem topologically equivalent to a shift on two symbols. 
3.3. Mixing in the perturbed cat's eye flow. By exploiting the symmetry of this model, we see that this perturbing function breaks up all trajectories transversely. In fact, we can view both the perturbed and unperturbed cases as flows on the cylinder. Here we take $x \in \mathbb{R} / 2 \pi, y \in \mathbb{R}$. All of the saddle points are identified and we obtain two homoclinic orbits to a single saddle point. We can now use the standard homoclinic theorem to find a shift on two symbols.

Based on the proof of the theorem from the second section, we expect mixing to occur at least within the region around the fixed point. We know that there exists a neighborhood $U$ of the fixed point $(0,2 \pi N)$ on which the Poincare map for this system acts like a version of the horseshoe map (see Fig. 3.2).

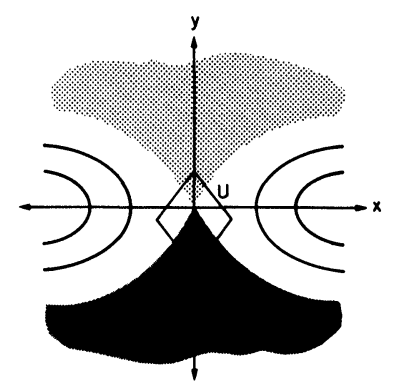

(a)

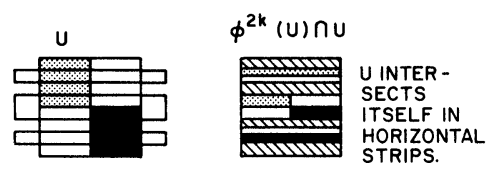

(b)

FIG. 3.2. (a) The cat's eye flow on the cylinder. (b) Perturbed cat's eye flow. Here the top and bottom layers are mixed into the cat's eyes region and eventually into each other.

Viewed as a flow on the plane, we see that the perturbed system has a geometric structure similar to that of Holmes's perturbed sine-Gordon equation $[11, \S 3]$. We show that the perturbed cat's eye flow has a subsystem isomorphic to the shift on the symbols "+" and "-," where the "+" corresponds to traveling "downstream" along an upper trajectory and the "-" corresponds to traveling "upstream" along a lower trajectory (see Fig. 3.3). This provides a mechanism for fluid inside one cat's eye to travel both upstream and downstream. This mechanism does not exist for the unperturbed case, since flow within an "eye" will remain there for all time. In the perturbed system, all saddle connections are broken up to give us transversal intersection of stable and unstable manifolds. The heteroclinic theorem tells us that at each fixed point $p_{n}=(2 \pi n, 0)$, there is a neighborhood $U_{n}$, a unit square in local coordinates, such that for some fixed time $T^{*}$, the flow $\varphi_{T^{*}}$ maps $U_{i}$ to intersect $U_{i-1}$ and $U_{i+1}$ in horizontal strips. A simplified model of the dynamics present is pictured in Fig. 3.4. Here each $U_{i}$ is intersected by the horizontal strips $H_{i-1, i}=\varphi\left(U_{i-1}\right) \cap U_{i}$ and $H_{i+1, i}=$ $\varphi\left(U_{i+1}\right) \cap U_{i}$. 


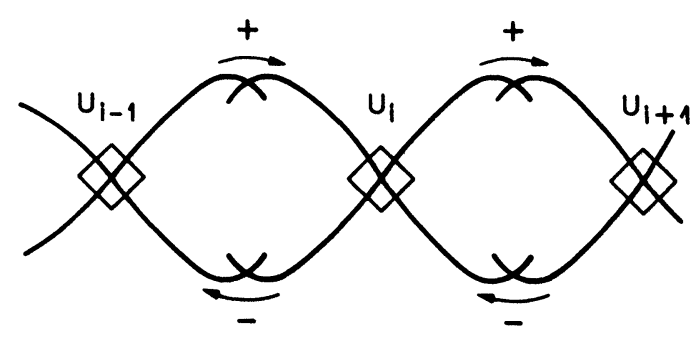

FIG. 3.3

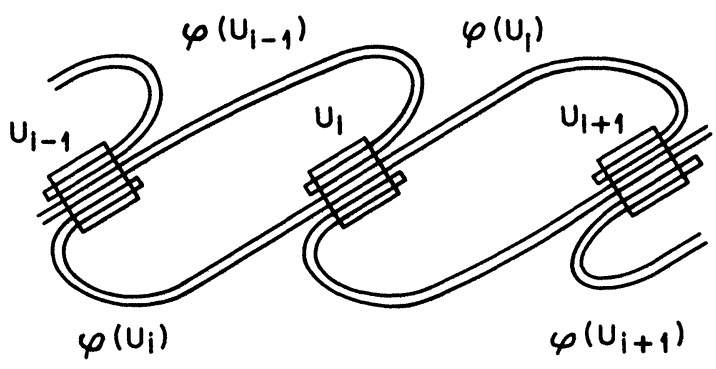

FIG. 3.4

By the symmetry of the flow and its perturbation, we can choose each $U_{i}$ so that $U_{i}+2 \pi=U_{i+1}$ and $\varphi\left(U_{i}\right)+2 \pi=\varphi\left(U_{i+1}\right)$. Our invariant set is

$$
I=\bigcap_{k=-\infty}^{\infty} \varphi^{-k}\left(\bigcup_{i}\left(H_{i+1, i} \cup H_{i-1, i}\right)\right) .
$$

$I$ can be decomposed into disjoint sets $I_{i}=U_{i} \cap I$. For any given $i$, we have a one-to-one correspondence between $I_{i}$ and $S^{ \pm}$, the set of all bi-infinite sequences of "+" and " - ":

$$
\begin{aligned}
& \tau: I_{i} \rightarrow S^{ \pm}, \\
& \begin{aligned}
{[\tau(x)]_{l}=} & + \text { if } \varphi^{\prime}(x) \in U_{k} \Rightarrow \varphi^{l+1}(x) \in U_{k+1}, \\
= & - \text { if } \varphi^{l}(x) \in U_{k} \Rightarrow \varphi^{l+1}(x) \in U_{k-1} .
\end{aligned}
\end{aligned}
$$

Thus there is a set $S^{ \pm}$of sequences corresponding to each $I_{i}$. We see that there is a mechanism for pieces of fluid to move rather chaotically both upstream and downstream as well as for fluid within each "eye" to mix with fluid in other "eyes." This mixing and chaotic motion was not present in the unperturbed cat's eye flow. The fact that the perturbation cos $k t$ leads to such chaos for almost all $k$ indicates that such mixing may be rather common in the actual shear layers.

4. Planar lattice flow. We consider the following flow:

$$
\dot{x}_{1}=-\sin \left(2 \pi x_{1}\right) \sin \left(2 \pi x_{2}\right), \quad \dot{x}_{2}=-\cos \left(2 \pi x_{1}\right) \cos \left(2 \pi x_{2}\right)
$$

a Hamiltonian system with $H_{0}=(2 \pi)^{-1} \sin \left(2 \pi x_{1}\right) \cos \left(2 \pi x_{2}\right)$ (see Fig. 4.1). This is a model for axisymmetric Taylor vortex flow as well as for many convective flows. If we take $x_{1}$ to be a moving coordinate, these equations model the Rossby waves of geophysical fluid dynamics (see [24, p. 84]). This flow is obviously doubly periodic, yielding a flow on the torus $T=\mathbb{R}^{2} / \Gamma$ where $\Gamma$ is the lattice $\left\{\left(n_{1}, n_{2}\right) ; n_{1}, n_{2} \in \mathbb{Z}\right\}$.

Viewed as a flow on the torus $T$, we obtain a system with heteroclinic orbits connecting four saddle points. Melnikov's theory can then be applied to perturbations of this flow. 


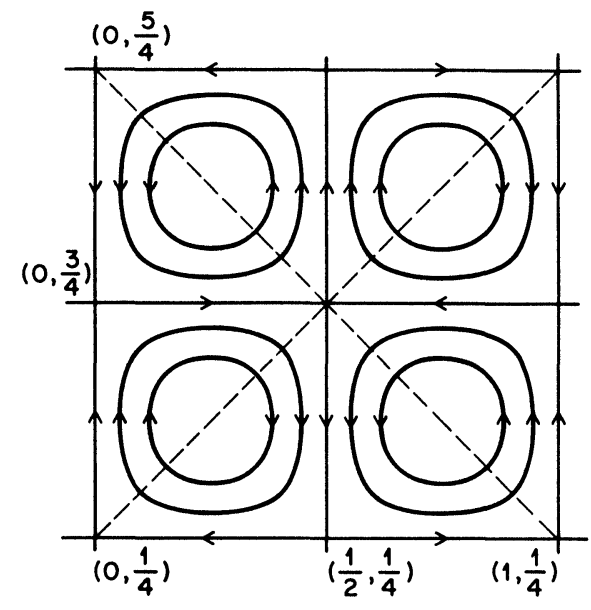

FIG. 4.1. $\Gamma^{\prime}$ is represented by the dashed line.

We can also map this flow onto a "smaller" torus $T^{\prime}=\mathbb{R}^{2} / \Gamma^{\prime}$ where $\Gamma^{\prime}=\left\{\left(\frac{1}{2}\left(n_{1}-n_{2}\right)\right.\right.$, $\left.\left.\frac{1}{2}\left(n_{1}+n_{2}\right)\right)\right\}$ (see Fig. 4.1). Here we have exploited the periodicity in the variables $\left(x_{1}-x_{2}\right),\left(x_{1}+x_{2}\right)$ as well as in $x_{1}$ and $x_{2}$. The flow on $T^{\prime}$ has only two heteroclinic saddle points. By examining perturbed flows on $T^{\prime}$, we can look for a subsystem that is a shift on two symbols. This horseshoe-like structure will result if all heteroclinic orbits are broken up so that stable and unstable manifolds intersect transversely.

4.1. Time- and space-dependent perturbations. We consider two types of perturbations, ones that are functions of time only and ones that have an added space dependence. In the purely time-dependent case, we have $\varepsilon \vec{f}(t)$ as a perturbation to the velocity field, with $\vec{f}(t)=\vec{f}(t+T)$. This corresponds to an external driving force $\vec{F}_{\varepsilon}=\varepsilon \vec{f}^{\prime}(t)$ that is uniform in space at any given moment. This is physically reasonable as an approximation to an external force that is time-periodic and has an average space variation much larger than the periodic lattice structure of the flow. For the vertical saddle connections, the Melnikov function for this perturbation is

$$
M_{v}\left(t_{0}\right)= \pm \int_{-\infty}^{\infty} \cos \left(2 \pi x_{2}(t)\right) f_{1}\left(t+t_{0}\right) d t
$$

since $\sin \left(2 \pi x_{1}\right)=0$ for these trajectories. Likewise for the horizontal orbits, $\cos \left(2 \pi x_{2}\right)=$ 0 and so

$$
M_{h}\left(t_{0}\right)= \pm \int_{-\infty}^{\infty} \sin \left(2 \pi x_{1}(t)\right) f_{2}\left(t+t_{0}\right) d t
$$

We see that the vertical and horizontal components of $\vec{f}$ are decoupled. We will show by symmetry properties that $f_{1}$ and $f_{2}$ must satisfy the same conditions in order for $M_{v}$ and $M_{h}$ to have simple zeros. For this space-independent perturbation, the $\Gamma^{\prime}$-lattice symmetry is preserved and chaotic motion can be reduced to a subsystem isomorphic to the shift on two symbols. The following example presents a spatially dependent perturbation that breaks up the $\Gamma^{\prime}$ symmetry.

In general, a perturbing velocity of the form

$$
\varepsilon\left(\begin{array}{l}
v_{1}\left(x_{2}, t\right) \\
v_{2}\left(x_{1}, t\right)
\end{array}\right)
$$


constitutes a solution to the two-dimensional Euler equation with external force

$$
\vec{F}_{\varepsilon}=\varepsilon\left(\begin{array}{l}
\partial v_{1}\left(x_{2}, t\right) / \partial t \\
\partial v_{2}\left(x_{1}, t\right) / \partial t
\end{array}\right)
$$

A particularly interesting perturbation of this form is

$$
\varepsilon\left(\begin{array}{l}
v_{1} \\
v_{2}
\end{array}\right)=\varepsilon\left(\begin{array}{l}
\cos \left(2 \pi x_{2}\right) \cos k t \\
\sin \left(2 \pi x_{1}\right) \cos k t
\end{array}\right)
$$

This has a stream function

$$
\frac{\varepsilon}{2 \pi} \cos k t\left[\sin \left(2 \pi x_{2}\right)-\cos \left(2 \pi x_{1}\right)\right]
$$

which can be viewed as a superposition of linear waves traveling along coordinate axes:

$$
-\left(e^{i\left(2 \pi x_{1}+k t\right)}+e^{i\left(2 \pi x_{1}-k t\right)}\right)-i\left(e^{i\left(2 \pi x_{2}+k t\right)}-e^{i\left(2 \pi x_{2}-k t\right)}\right) .
$$

This perturbation is geometrically interesting because it breaks up the $\Gamma^{\prime}$ symmetry and we are forced to consider heteroclinic orbits joining four points instead of two points. We shall show that for almost all $k$, the saddle connections break up to yield a subsystem topologically equivalent to the shift on four consecutive symbols.

4.2. Explicit calculation of the Melnikov functions. Along an unperturbed horizontal saddle connection, we have

$$
\dot{x}_{1}= \pm \sin \left(2 \pi x_{1}\right), \quad \dot{x}_{2}=0
$$

and along a vertical connection

$$
\dot{x}_{2}= \pm \cos \left(2 \pi x_{2}\right), \quad \dot{x}_{1}=0 .
$$

In the case of the connection from $\left(\frac{1}{2}, \frac{1}{4}\right)$ to $\left(0, \frac{1}{4}\right)$, we have $\dot{x}_{1}=-\sin \left(2 \pi x_{1}\right)$. This has a solution $x_{1}=(1 / \pi) \tan ^{-1}\left(e^{-2 \pi t}\right)$, which by symmetry properties of the flow yields

$$
\sin \left(2 \pi x_{1}\right)= \pm \frac{2 e^{-2 \pi t}}{1+e^{-4 \pi t}}
$$

along all horizontal connections and

$$
\cos \left(2 \pi x_{2}\right)= \pm \frac{2 e^{-2 \pi t}}{1+e^{-4 \pi t}}
$$

along all vertical ones.

For a spatially independent perturbation, the Melnikov function of $\S 4.1$, for either saddle connection, is of the form

$$
M_{i}\left(t_{0}\right)=\int_{-\infty}^{\infty} \frac{2 e^{-2 \pi t}}{1+e^{-4 \pi t}} f_{i}\left(t+t_{0}\right) d t
$$

If we expand $f_{i}$ into its Fourier series

$$
f_{i}(t)=\sum_{k=-\infty}^{\infty}\left(A_{k} \cos \left(\frac{2 \pi k t}{T}\right)+B_{k} \sin \left(\frac{2 \pi k t}{T}\right)\right)
$$

we find that

$$
M_{i}\left(t_{0}\right)=\sum_{k=-\infty}^{\infty}\left[\left(A_{k} \cos \left(\frac{2 \pi k t_{0}}{T}\right)+B_{k} \sin \left(\frac{2 \pi k t_{0}}{T}\right)\right) \int_{-\infty}^{\infty} \frac{2 e^{-2 \pi t}}{1+e^{-4 \pi t}} \cos \left(\frac{2 \pi k t}{T}\right) d t\right] .
$$


Evaluation by residues reveals

$$
M_{i}\left(t_{0}\right)=\sum_{k=-\infty}^{\infty}\left(A_{k} \cos \left(\frac{2 \pi k t_{0}}{T}\right)+B_{k} \sin \left(\frac{2 \pi k t_{0}}{T}\right)\right)\left(\frac{1}{e^{-\pi k / 2 T}+e^{\pi k / 2 T}}\right) .
$$

Whether or not $M_{i}\left(t_{0}\right)$ has simple zeros depends on the respective values of $A_{k}$ and $B_{k}$. For example, if $f_{i}=A_{0}+A_{1} \cos (2 \pi k t / T)$, we require

$$
\left|A_{0}\right|<\left|A_{1}\right|\left[\frac{2}{e^{-\pi k / 2 T}+e^{\pi k / 2 T}}\right]
$$

for $M_{i}\left(t_{0}\right)$ to have simple zeros. Now we see that the class of perturbing functions $\vec{f}=(A \cos (t), B \sin (t))$ yields $M_{v}\left(t_{0}\right)$ and $M_{h}\left(t_{0}\right)$ with simple zeros for all saddle connections. Applying the results of $\S 2$, we obtain a shift on four symbols as a subsystem of the perturbed flow on $T$, and a shift on two symbols as a subsystem of the perturbed flow on $T^{\prime}$.

For the spatially dependent perturbation

$$
\varepsilon\left(\begin{array}{c}
\cos \left(2 \pi x_{2}\right) \cos k t \\
\sin \left(2 \pi x_{1}\right) \cos k t
\end{array}\right)
$$

we find that, up to a change of sign, the Melnikov function for either a vertical or horizontal saddle connection is

$$
M\left(t_{0}\right)=4 \cos \left(k t_{0}\right) \int_{-\infty}^{\infty} \frac{e^{-4 \pi t}}{\left(1+e^{-4 \pi t}\right)^{2}} \cos k t d t
$$

which we evaluate via residues (using the procedure outlined in Appendix A for the calculation of the integral in $\S 3$ ) to be

$$
\begin{aligned}
M\left(t_{0}\right) & =\cos k t_{0}\left(\frac{k}{4 \pi \sinh (k / 4)}\right) \\
& =\cos k t_{0} M_{0}(k) .
\end{aligned}
$$

$M_{0}(k)$ is nonzero for almost all $k$ so that the Melnikov function will have simple zeros and we have a subsystem topologically equivalent to the shift on four consecutive symbols.

4.3. Mixing in the perturbed lattice flow. Under both perturbations, we expect some sort of mixing to occur that was not present in the unperturbed case. In the perturbed systems, all connections are broken up to yield transverse intersection of stable and unstable manifolds. As in the cat's eye model, at each fixed point $p_{n_{1} n_{2}}=$ $\left(\frac{1}{2} n_{1}, \frac{1}{2} n_{2}+\frac{1}{4}\right), n_{1}, n_{2} \in \mathbb{Z}$, we have neighborhoods $U_{n_{1} n_{2}}$ that intersect each other in horizontal strips under some fixed time mapping of the flow (see Fig. 4.2). In the case of the first perturbation studied, we can exploit the $T^{\prime}$ symmetry to obtain a subsystem topologically equivalent to the shift on two symbols. The perturbed and unperturbed systems are both flows on the torus $T^{\prime}$. Under this symmetry, we can identify all clockwise rotating cells with each other and likewise all counterclockwise rotating cells with each other (Fig. 4.3). In the unperturbed case, these patches of fluid do not mix. The perturbation satisfies the conditions of the heteroclinic theorem with two fixed points, yielding a subsystem of the flow topologically equivalent to the shift on two symbols. In the perturbed case we see mixing patterns similar to those present in the cat's eye flow.

In the case of the second perturbation, we do not have the $T^{\prime}$ symmetry. The cells break up into two different clockwise and counterclockwise rotations (see Fig. 4.3). 


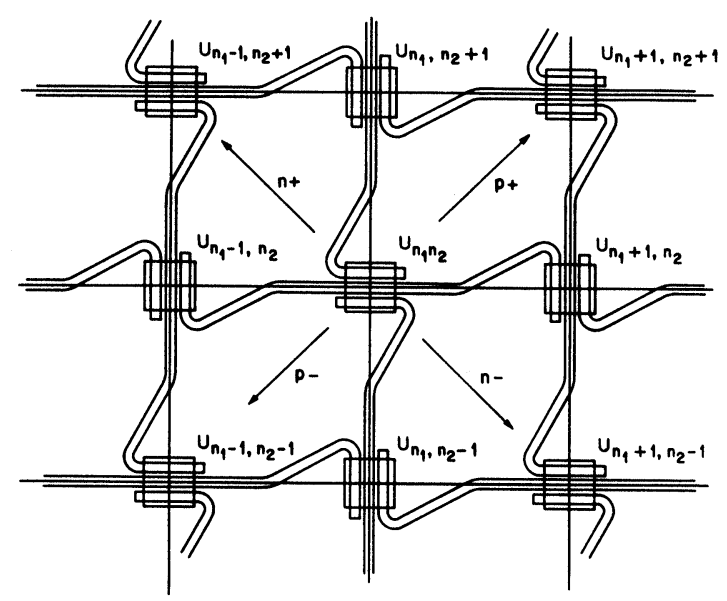

FIG. 4.2

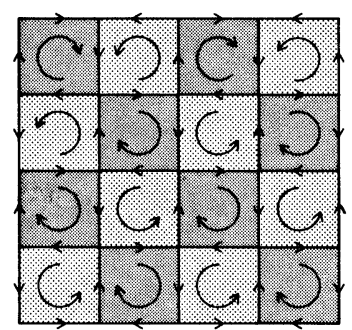

(a)

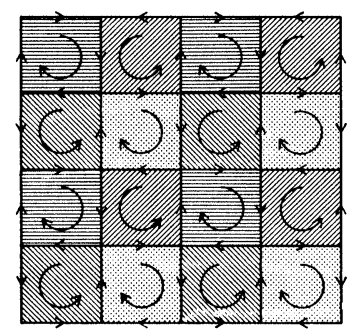

(b)

FIG. 4.3. (a) Flow on $T^{\prime}$. All clockwise rotating cells are identified, as are all counterclockwise rotating cells. (b) Flow on T. There are two types of clockwise rotations as well as two types of counterclockwise rotations.

On the torus $T$ we have four fixed points in the heteroclinic orbit and our system breaks up to yield a subsystem topologically equivalent to the shift on four consecutive symbols. In the previous case we have symbols 1 and 2 identified with 3 and 4 , respectively. This is analogous to identifying the two clockwise rotations with each other and likewise the two counterclockwise rotations with each other. Again we expect similar mixing patterns to occur.

In the cat's eye flow, we found a mechanism for traveling up- and downstream randomly within the cat's eyes. This corresponded to a shift in the symbols "+" and "-." In the lattice flow, we find a mechanism for traveling all over the plane, along the $\Gamma^{\prime}$ lattice. We find that the perturbed lattice flow has a subsystem isomorphic to the shift on the four symbols " $n_{+}$, " " $n_{-}$, , " $p_{+}$, , " $p_{-}$." Here, $n_{ \pm}$corresponds to a 
translation by $\pm\left(-\frac{1}{2}, \frac{1}{2}\right)$ along the lattice. Likewise $p_{ \pm}$corresponds to a translation by $\pm\left(\frac{1}{2}, \frac{1}{2}\right)$ (see Fig. 4.2).

In the neighborhood $U_{n_{1} n_{2}}$ of each fixed point $p_{n_{1} n_{2}}$, we find that for some fixed time $T^{*}$, the flow $\varphi_{T^{*}}$ maps $U_{n_{1} n_{2}}$ to intersect $U_{n_{1}-1, n_{2}}$ and $U_{n_{1}+1, n_{2}}$ for $n_{1}+n_{2}$ odd, or $U_{n_{1}, n_{2}-1}$ and $U_{n_{1}, n_{2}+1}$ for $n_{1}+n_{2}$ even, in horizontal strips (see Fig. 4.2).

These strips are mapped to smaller strips in $U_{n_{1}-1, n_{2}-1}, U_{n_{1}+1, n_{2}-1}, U_{n_{1}-1, n_{2}+1}$, $U_{n_{1}+1, n_{2}+1}$, by a second iteration of $\varphi_{T^{*}}$. Thus $\varphi_{2 T^{*}}$ maps $U_{n_{1} n_{2}}$ to intersect $U_{n_{1}-1, n_{2}-1}$, $U_{n_{1}+1, n_{2}-1}, U_{n_{1}-1, n_{2}+1}, U_{n_{1}+1, n_{2}+1}$, in horizontal strips.

For convenience, we now refer to $\varphi_{2 T^{*}}$ as $\varphi$. Thus our invariant set is

$$
I=\bigcap_{k=-\infty}^{\infty} \varphi^{-k}\left(\bigcup_{n_{1}, n_{2}}\left(\varphi\left(U_{n_{1} n_{2}}\right) \cap\left(U_{n_{1}-1, n_{2}-1} \cup U_{n_{1}+1, n_{2}-1} \cup U_{n_{1}-1, n_{2}+1} \cup U_{n_{1}+1, n_{2}+1}\right)\right)\right),
$$

where $I$ can be decomposed into the disjoint sets $I_{n_{1} n_{2}}=U_{n_{1} n_{2}} \cap I$. For any given pair $\left(n_{1}, n_{2}\right)$, there is a one-to-one correspondence between $I_{n_{1} n_{2}}$ and the set of bi-infinite sequences on the symbols $p_{+}, p_{-}, n_{+}, n_{-}$:

$$
\begin{aligned}
& \tau: I_{n_{1} n_{2}} \rightarrow S \\
& {[\tau(x)]_{l}=p_{+} \quad \text { if } \varphi^{l}(x) \in U_{n_{1} n_{2}} \Rightarrow \varphi^{l+1}(x) \in U_{n_{1}+1, n_{2}+1},} \\
& {[\tau(x)]_{l}=p_{-} \quad \text { if } \varphi^{l}(x) \in U_{n_{1} n_{2}} \Rightarrow \varphi^{l+1}(x) \in U_{n_{1}-1, n_{2}-1},} \\
& {[\tau(x)]_{l}=n_{+} \quad \text { if } \varphi^{l}(x) \in U_{n_{1} n_{2}} \Rightarrow \varphi^{l+1}(x) \in U_{n_{1}-1, n_{2}+1},} \\
& {[\tau(x)]_{l}=n_{-} \quad \text { if } \varphi^{l}(x) \in U_{n_{1} n_{2}} \Rightarrow \varphi^{l+1}(x) \in U_{n_{1}+1, n_{2}-1} .}
\end{aligned}
$$

Thus, fluid particles within one cell can travel randomly around the plane in the perturbed case. In the unperturbed case, this sort of mixing is not allowed since fluid within one cell will remain there for all time.

5. Motion of an elliptical vortex in a strain field. An important part of fluid mechanics is the study of vortices, their structure, and how they interact with one another. In $\S \S 3$ and 4 we examined two well-known two-dimensional planar fluid models. Since organized vortex structures are observed frequently, we would like to find a simple model for a vortex affected by a field of neighboring vortices. As the examples of $\S \S 3$ and 4 indicate, the presence of multiple vortices in stationary planar fluid flow often results in fixed points of the flow, between vortex structures, that can be modeled as hyperbolic saddle points in a planar dynamical system. In a neighborhood of such saddle points, the velocity field is roughly linear and can be locally approximated by a simple strain. Thus it is physically reasonable to model certain vortex interaction locally as a single vortex in a straining flow. Moore and Saffman [20], as well as Neu [21], describe vortex interaction that can be modeled in such a way.

We study the motion of an elliptical vortex in a three-dimensional imposed strain. We see that the evolution of such a vortex can be characterized as a planar dynamical system that has interesting Hamiltonian and non-Hamiltonian formulations involving the aspect ratio $\eta=a / b$ and the angle $\theta$ of rotation of the ellipse. Here $a$ and $b$ correspond to the major and minor axes of the ellipse. We apply Melnikov's method to the evolution equations of the vortex to show chaotic dynamics occurring in the presence of three-dimensional periodic stretching of the imposed strain. The actual analysis differs somewhat from what was done in the previous sections in that we study chaos occurring in the evolution equation of the shape and orientation of the ellipse as opposed to chaos occurring in the flow pattern of an actual fluid model. 
5.1. Hamiltonian formulation of exact Euler solution. The Hamiltonian formulation presented below is due to Neu [22] and represents a three-dimensional generalization of the exact solutions of an elliptical vortex in a two-dimensional straining flow (described by Kida [14]). First consider a planar vortex region in the shape of an ellipse with constant vorticity in the interior. The points on the boundary of the region are solutions to the equation $x^{2} / a^{2}+y^{2} / b^{2}=$ constant. Following a potential theory calculation described in Lamb [15], we see that the velocity field inside the ellipse is linear:

$$
\begin{gathered}
\left(\begin{array}{c}
\dot{x} \\
\dot{y}
\end{array}\right)=\tilde{U}(a, b, \theta)\left(\begin{array}{l}
x \\
y
\end{array}\right), \\
\tilde{U}(a, b, \theta)=-\frac{\omega}{a+b} R(\theta)\left(\begin{array}{cc}
0 & a \\
-b & 0
\end{array}\right) R(\theta) .
\end{gathered}
$$

Here $a$ and $b$ correspond, respectively, to the major and minor axes of this elliptical cross-section and $\theta$ is the angle of the major axis with respect to the $x$-axis. $R(\theta)$ is the rotation matrix

$$
\left(\begin{array}{rr}
\cos \theta & -\sin \theta \\
\sin \theta & \cos \theta
\end{array}\right)
$$

In three dimensions we have a cylindrical vortex region whose cross-section in the $x y$-plane is the above velocity field. We add an irrotational straining field the velocity of which is given by $v=\left(\gamma^{\prime} x,-\gamma y, \gamma^{\prime \prime} z\right)$ where $\gamma^{\prime}-\gamma+\gamma^{\prime \prime}=0$ is required for incompressibility. The combination of vortex and strain yields a fluid velocity that, in the $x y$-plane, has the form $U(a, b, \theta)(x, y)^{T}$ where

$$
U(a, b, \theta)=-\frac{\omega}{a+b} R(\theta)\left(\begin{array}{cc}
0 & a \\
-b & 0
\end{array}\right) R(\theta)+\left(\begin{array}{cc}
\gamma^{\prime} & 0 \\
0 & -\gamma
\end{array}\right)
$$

The velocity field inside the vortex is again linear and the path of a fluid particle on the boundary must satisfy the equation of an ellipse which we write in matrix form:

$$
\begin{gathered}
\left(\begin{array}{l}
x \\
y
\end{array}\right) E(a, b, \theta)\left(\begin{array}{ll}
x & y
\end{array}\right)=\text { constant, } \\
E(a, b, \theta)=R(\theta)\left(\begin{array}{cc}
a^{-2} & 0 \\
0 & b^{-2}
\end{array}\right) R(\theta) .
\end{gathered}
$$

Differentiating the ellipse equation, we obtain

$$
\dot{X}^{T} E X+X^{T} \dot{E} X+X^{T} E \dot{X}=0
$$

where $X$ is the vector $(x, y)$. Since $\dot{X}=U(a, b, \theta) X$, we have the matrix evolution equation

$$
\dot{E}+U^{T} E+E U=0
$$

which we can write out explicitly in terms of $a, b$, and $\theta$ to give us the evolution equations for the elliptical vortex:

$$
\begin{gathered}
\dot{a}+\left(\gamma \sin ^{2} \theta-\gamma^{\prime} \cos ^{2} \theta\right) a=0, \\
\dot{b}+\left(\gamma \cos ^{2} \theta-\gamma^{\prime} \sin ^{2} \theta\right) b=0, \\
\dot{\theta}=\frac{\omega a b}{(a+b)^{2}}-\frac{1}{2}\left(\gamma+\gamma^{\prime}\right) \frac{a^{2}+b^{2}}{a^{2}-b^{2}} \sin 2 \theta .
\end{gathered}
$$

These evolution equations have the following Hamiltonian formulation: let $\eta=a / b$ be the aspect ratio and $\tau$ be a dimensionless time defined by $d \tau / d t=\omega \eta^{2} /\left(\eta^{2}-1\right)$. 
Then the evolution equations become

$$
\begin{aligned}
& \frac{d \eta}{d \tau}=-\frac{\partial H}{\partial \theta}=\frac{\gamma+\gamma^{\prime}}{\omega}\left(\eta-\frac{1}{\eta}\right) \cos 2 \theta \\
& \frac{d \theta}{d \tau}=\frac{\partial H}{\partial \eta}=\frac{\eta-1}{\eta(1+\eta)}-\frac{1}{2} \frac{\gamma+\gamma^{\prime}}{\omega}\left(1+\frac{1}{\eta^{2}}\right) \sin 2 \theta \\
& H=\log \frac{(1+\eta)^{2}}{\eta}-\frac{1}{2} \frac{\gamma+\gamma^{\prime}}{\omega}\left(\eta-\frac{1}{\eta}\right) \sin 2 \theta .
\end{aligned}
$$

We consider $\gamma, \gamma^{\prime}$, and $\omega$ to be, in general, time-dependent parameters in this equation. The total circulation of the vortex is $\Gamma=\pi a b \omega$, which we know to be constant by the Kelvin Circulation Theorem (see [6, p. 28]). The evolution equations imply that

$$
\frac{d(a b)}{d t}=\left(\gamma^{\prime}-\gamma\right) a b
$$

so that

$$
a b=a_{0} b_{0} e^{\left(\gamma^{\prime}-\gamma\right) t}
$$

which in turn yields

$$
\omega=\omega_{0} e^{\left(\gamma-\gamma^{\prime}\right) t}
$$

Thus, when $\gamma^{\prime \prime}=0, \gamma^{\prime}-\gamma+\gamma^{\prime \prime}=0$ implies that $\gamma=\gamma^{\prime}$. Our Hamiltonian system is autonomous if and only if $\gamma^{\prime \prime}=0, \gamma, \gamma^{\prime}$ are both constant. We will consider the case where this autonomous Hamiltonian system is perturbed by a periodic stretching of the strain where we set $\gamma^{\prime \prime}=\varepsilon g(t)$.

In the autonomous case, we have $\gamma=\gamma^{\prime}$, and are interested in the dynamics indicated in the phase portrait for $0<\gamma / \omega<0.15$ (see Fig. 5.1). There are no heteroclinic

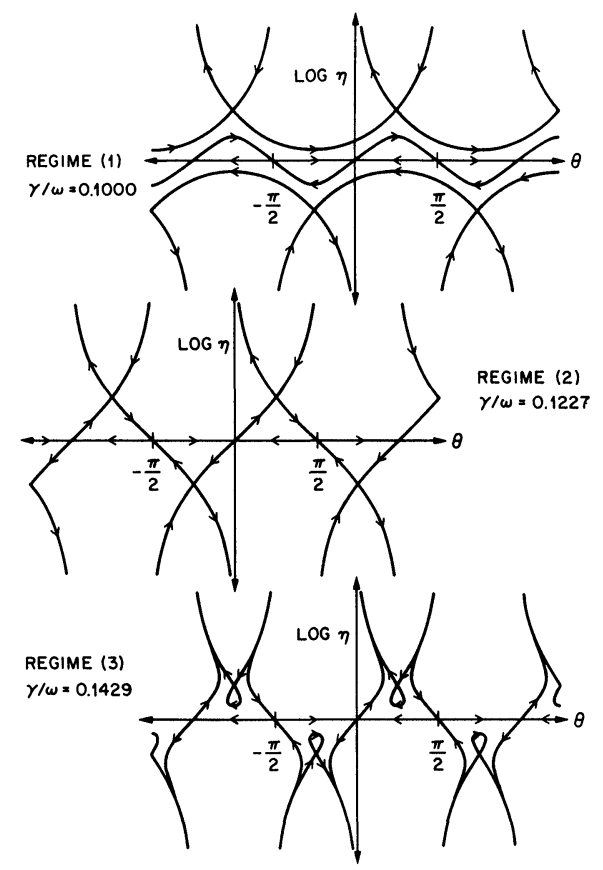

FIG. 5.1 
orbits in the phase portrait for $\gamma / \omega>.15$. The three interesting regimes are depicted in Fig. 5.1:

(1) For $0<\gamma / \omega<.1227$, there are oscillating regions (bubbles close to the $\log \eta=0$ axis) as well as rotating regions between the bubbles and the outer saddle connections.

(2) At $\gamma / \omega=.1227$ we have a bifurcation where saddle connections between three fixed points exist for this value of $\gamma / \omega$ only.

(3) For $\gamma / \omega$ between .1227 and .15 , we have homoclinic saddle connections, the interior of which represents an ellipse oscillating about the ray $\theta=\pi / 4$.

The importance of the bifurcation is that in regimes (2) and (3) we no longer have the possibility of a rotating ellipse.

5.2. Real time formulation of evolution equations. In order to apply Melnikov's method to the above Hamiltonian system, we would need to consider time-periodic perturbations of the dimensionless time $\tau$. This is not a reasonable physical model, since a periodic perturbation of the straining flow would be periodic in real time, and not in the dimensionless time $\tau$. Note that the evolution equations written in terms of the orientation, aspect ratio and real time are

$$
\begin{gathered}
\frac{d \theta}{d t}=\frac{\omega \eta}{(\eta+1)^{2}}-\frac{1}{2}\left(\gamma+\gamma^{\prime}\right) \frac{\eta^{2}+1}{\eta^{2}-1} \sin 2 \theta, \\
\frac{d \eta}{d t}=\left(\gamma+\gamma^{\prime}\right) \eta \cos 2 \theta .
\end{gathered}
$$

Since $(\eta, \theta)$ and $\left(\eta^{-1}, \theta+\pi / 2\right)$ correspond to the same ellipse, we can parameterize the evolution equation in terms of $r=\log \eta, \varphi=2 \theta$, and yield a polar coordinates formulation for these equations in which there is a one-to-one correspondence between ellipses and points in the phase space $(r, \varphi)$. The evolution equations become

$$
\begin{gathered}
\dot{r}=\left(\gamma+\gamma^{\prime}\right) \cos \varphi \\
\dot{\varphi}=\frac{2 \omega e^{r}}{\left(e^{r}+1\right)^{2}}-\left(\gamma+\gamma^{\prime}\right) \frac{e^{2 r}+1}{e^{2 r}-1} \sin \varphi .
\end{gathered}
$$

From the Hamiltonian formulation, we know that trajectories correspond to constants of

$$
H=\log \left[\frac{\left(1+e^{r}\right)^{2}}{e^{r}}\right]-\frac{\gamma+\gamma^{\prime}}{2 \omega}\left(e^{r}-e^{-r}\right) \sin \varphi
$$

This can be verified by calculating $d H / d t=0$ for the real time $t$. These equations seem to blow up for $r=0$. Fortunately, we see that this blow-up is due to the coordinates we are using and not the equations themselves. Polar coordinates are not well defined at $r=0$ so we convert the equations to Cartesian form by $x=r \cos \varphi, y=r \sin \varphi$. The evolution equations become:

$$
\begin{aligned}
& \dot{x}=\left(\gamma+\gamma^{\prime}\right) \frac{x^{2}}{x^{2}+y^{2}}-\frac{2 \omega y e^{\sqrt{x^{2}+y^{2}}}}{\left(e^{\sqrt{x^{2}+y^{2}}}+1\right)^{2}}+\left(\gamma+\gamma^{\prime}\right) \frac{e^{2 \sqrt{x^{2}+y^{2}}}+1}{e^{2 \sqrt{x^{2}+y^{2}}}-1} \frac{y^{2}}{\sqrt{x^{2}+y^{2}}}, \\
& \dot{y}=\left(\gamma+\gamma^{\prime}\right) \frac{x y}{x^{2}+y^{2}}+\frac{2 \omega x e^{\sqrt{x^{2}+y^{2}}}}{\left(e^{\sqrt{x^{2}+y^{2}}}+1\right)^{2}}-\left(\gamma+\gamma^{\prime}\right) \frac{e^{2 \sqrt{x^{2}+y^{2}}}+1}{e^{2 \sqrt{x^{2}+y^{2}}}-1} \frac{x y}{\sqrt{x^{2}+y^{2}}} .
\end{aligned}
$$

We see that as $r \rightarrow 0$, the first and third terms in $\dot{x}$ appear to blow up. Using Taylor 

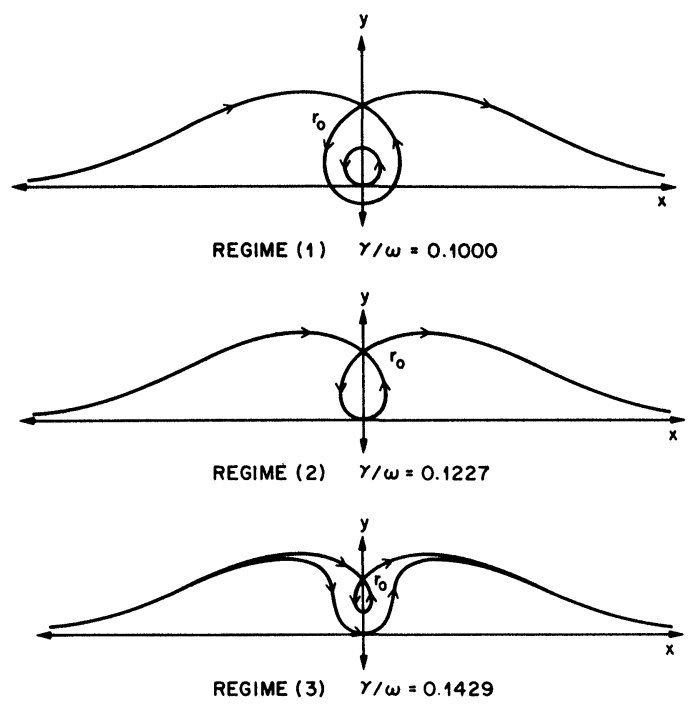

FIG. 5.2

expansion techniques, we see that the third term can be approximated by

$$
\left(\gamma+\gamma^{\prime}\right) \frac{y^{2}}{x^{2}+y^{2}}\left(1+\mathcal{O}\left(r^{2}\right)\right)
$$

for $r$ small. Thus, $\dot{x} \rightarrow \gamma+\gamma^{\prime}$ as $r \rightarrow 0$. In a similar fashion, we see that $\dot{y} \rightarrow 0$ as $r \rightarrow 0$.

The phase portrait (Fig. 5.2) for the real time formulation has a much simpler form than that of the Hamiltonian one we first introduced (Fig. 5.1). We see that for $\left(\gamma+\gamma^{\prime}\right) / 2 \omega<.15$, there is a homoclinic loop with hyperbolic fixed point corresponding to the largest root of $e^{r}\left(e^{r}-1\right)=\left(\left(\gamma+\gamma^{\prime}\right) / 2 \omega\right)\left(e^{2 r}+1\right)\left(e^{r}+1\right)$. We see that the bifurcation at $\gamma / \omega=.1227$ is represented by the loop crossing the origin.

5.3. Periodic stretching of an elliptical vortex. In general, our perturbed system will have the form

$$
\begin{gathered}
\dot{r}=C_{0} \cos \varphi+\varepsilon g_{1}(r, \varphi, t), \\
\dot{\varphi}=\frac{2 \omega_{0} e^{r}}{\left(e^{r}+1\right)^{2}}-C_{0} \frac{e^{2 r}+1}{e^{2 r}-1} \sin \varphi+\varepsilon g_{2}(r, \varphi, t) .
\end{gathered}
$$

Here $g_{1}$ and $g_{2}$ are periodic in time, $C_{0}=\left(\gamma_{0}+\gamma_{0}^{\prime}\right)$.

For $0<C_{0} / \omega_{0}<0.15$, the unperturbed system has a hyperbolic fixed point $p_{0}$ at $\varphi=\pi / 2, r=r_{0}$ where $r_{0}$ corresponds to the largest real root of the cubic $e^{3 r}+e^{2 r}(1-B)+e^{r}(1+B)+1=0$ where $B=\left(\frac{1}{2} C_{0} \omega_{0}\right)^{-1}$. This fixed point has a homoclinic saddle connection $\Gamma_{0}$ as depicted in Fig. 5.2. If we consider a perturbation involving a periodic stretching by an amount $\varepsilon \gamma^{\prime \prime}(t)$, then our perturbation has the form

$$
\begin{gathered}
g_{1}=C_{1}(t) \cos \varphi \\
g_{2}=\frac{2 C_{2}(t) e^{r}}{\left(e^{r}+1\right)^{2}}-C_{1}(t) \frac{e^{2 r}+1}{e^{2 r}-1} \sin \varphi .
\end{gathered}
$$

Here $C_{1}$ and $C_{2}$ are periodic in time with period $T$. We consider the symmetric case where the oscillation of $\gamma^{\prime \prime}$ puts equal and opposite oscillations on $\gamma^{\prime}$ and $\gamma$ while 
maintaining the incompressibility condition $\gamma^{\prime}-\gamma+\gamma^{\prime \prime}=0$. Thus, $\gamma+\gamma^{\prime}$ stays constant even though $\gamma-\gamma^{\prime}$ oscillates with $\gamma^{\prime \prime}$. This implies $C_{1}(t)=0$ so that our perturbation has the simpler form

$$
g_{1}=0, \quad g_{2}=\frac{2 C_{2}(t) e^{r}}{\left(e^{r}+1\right)^{2}}
$$

If we parameterize $\Gamma_{0}$ by $(r(t), \varphi(t))$, the Melnikov function for the perturbed system can be calculated using the non-Hamiltonian form. There are two ways of doing the Melnikov function calculation. We can view $\Gamma_{0}$ as a trajectory in the $(r, \varphi)$ coordinate system, which has the advantage of a simpler formulation. Since these coordinates break up at $r=0$, we cannot treat the case where $\Gamma_{0}$ contains the point $r=0$. This occurs only at the value $\gamma / \omega=.1227$. For any other value of $\gamma / \omega$, we can find a $C^{\infty}$ vector field $\left(f_{1}(r, \varphi), f_{2}(r, \varphi)\right)$ so that

$$
\dot{r}=f_{1}(r, \varphi), \quad \dot{\varphi}=f_{2}(r, \varphi)
$$

is a planar differentiable dynamical system in the coordinates $(r, \varphi)$ with a saddle connection identical to $\Gamma_{0}$ in its real time parameterization. We have $f_{1}=C_{0} \cos \varphi$, $f_{2}=2 \omega_{0} e^{r} /\left(e^{r}+1\right)^{2}-C_{0} \sin \varphi\left(e^{2 r}+1\right) /\left(e^{2 r}-1\right)$ in a neighborhood of the curve $\Gamma_{0}$. This new dynamical system is suitable for Melnikov's method and in a neighborhood of $\Gamma_{0}$ has dynamics identical to that of the original system.

Alternatively, we can treat the evolution equation as a dynamical system in the $(x, y)$ coordinates. This allows us to show that chaos will also occur in the degenerate case of $\gamma / \omega=.1227$. Both calculations are presented.

The Melnikov function in $(r, \varphi)$ coordinates. For this we need to know $\exp \left(\int_{0}^{t} \operatorname{tr} D f\left(\Gamma_{0}(s)\right) d s\right)$. We have

$$
\operatorname{tr} D f=-C_{0} \frac{e^{2 r}+1}{e^{2 r}-1} \cos \varphi=\frac{-\dot{r}\left(e^{2 r}+1\right)}{e^{2 r}-1} .
$$

This gives us

$$
\exp \left(\int_{0}^{t} \operatorname{tr} D f\left(\Gamma_{0}(s)\right) d s\right)=\frac{e^{r(t)}\left(e^{r_{0}}-e^{-r_{0}}\right)}{e^{2 r(t)}-1}
$$

This yields a Melnikov function

$$
\begin{gathered}
M\left(t_{0}\right)=C_{3} \int_{-\infty}^{\infty} \frac{e^{2 r} \cos \varphi}{\left(e^{r}+1\right)^{2}\left(e^{2 r}-1\right)} C_{2}\left(t+t_{0}\right) d t, \\
C_{3}=C_{0}\left(e^{r_{0}}-e^{-r_{0}}\right) .
\end{gathered}
$$

Using the fact that the integral represents a convolution with an odd function, for $C_{2}=\cos k t$, we have

$$
\begin{aligned}
M\left(t_{0}\right) & =C_{3} \sin k t_{0} \int_{-\infty}^{\infty} \frac{e^{2 r} \cos \varphi}{\left(e^{r}+1\right)^{2}\left(e^{2 r}-1\right)} \sin k t d t \\
& =\sin k t_{0} M_{0}(k) .
\end{aligned}
$$

Since $\cos \varphi \propto \dot{r}(t)$, we can see that the above integral is the sine transform of an $L^{1}$ function.

$$
\begin{aligned}
\int_{-\infty}^{\infty}\left|\frac{e^{2 r} \cos \varphi}{\left(e^{r}+1\right)^{2}\left(e^{2 r}-1\right)}\right| d t & =2 \int_{0}^{\infty}\left|\frac{e^{2 r} \cos \varphi}{\left(e^{r}+1\right)^{2}\left(e^{2 r}-1\right)}\right| d t \\
& \leqq \frac{C_{3}}{C_{0}} \int_{r(0)}^{r(\infty)} \frac{e^{2 r}}{\left(e^{r}+1\right)^{2}\left(e^{2 r}-1\right)} d r<\infty .
\end{aligned}
$$


We know by the properties of the Fourier transform on $L^{1}(\mathbb{R})([13, \mathrm{pp} .120-131])$ that $M_{0}(k)$ is a uniformly continuous function of $k$ that is not identically zero. Thus there exists some interval $k_{1} \leqq k \leqq k_{2}$ such that $M_{0}(k)$ is nonzero. For these values of $k$, $M\left(t_{0}\right)$ has simple zeros.

The Melnikov function in $(x, y)$ coordinates. We now consider the dynamical system

$$
\begin{gathered}
\dot{x}=\left(\gamma+\gamma^{\prime}\right) \cos ^{2} \varphi-\frac{2 \omega r \sin \varphi e^{r}}{\left(e^{r}+1\right)^{2}}+\left(\gamma+\gamma^{\prime}\right) \frac{e^{2 r}+1}{e^{2 r}-1} r \sin ^{2} \varphi, \quad r \neq 0, \\
\dot{y}=\left(\gamma+\gamma^{\prime}\right) \cos \varphi \sin \varphi+\frac{2 \omega r \cos \varphi e^{r}}{\left(e^{r}+1\right)^{2}}-\left(\gamma+\gamma^{\prime}\right) \frac{e^{2 r}+1}{e^{2 r}-1} r \sin \varphi \cos \varphi, \quad r \neq 0, \\
\dot{x}=\gamma+\gamma^{\prime}, \quad \dot{y}=0,
\end{gathered}
$$

for $r=0$. Here $r=\sqrt{x^{2}+y^{2}}, \varphi=\tan ^{-1}(y / x)$. We have the time-periodic perturbation

$$
\vec{g}(t, x, y)=\frac{\varepsilon C_{2}(t) r e^{r}}{\left(e^{r}+1\right)^{2}}\left(\begin{array}{c}
-\sin \varphi \\
\cos \varphi
\end{array}\right)
$$

The following analysis is for the case $\gamma+\gamma^{\prime}=.1227$; the nondegenerate case can be studied in a similar fashion. We have

$$
\begin{aligned}
f \wedge g & =\left(\gamma+\gamma^{\prime}\right) \cos \varphi C_{2}\left(t_{0}\right)\left(\frac{r e^{r}}{\left(e^{r}+1\right)^{2}}\right), \\
\operatorname{tr} D f & =\left(\gamma+\gamma^{\prime}\right) \cos \varphi\left(\frac{1}{r}-\frac{e^{2 r}+1}{e^{2 r}-1}\right) \quad r \neq 0, \\
& =0, \quad r=0, \\
e^{\int_{0}^{t} \operatorname{tr} D f d s} & =\frac{2 r(t) e^{r(t)}}{e^{2 r(t)}-1} .
\end{aligned}
$$

For $C_{2}(t)=\cos k t$, our Melnikov function is

$$
\begin{aligned}
M\left(t_{0}\right) & =-\int_{-\infty}^{\infty} \sin k t_{0} \dot{r}(t) \sin k t \frac{r^{2} e^{2 r}}{\left(e^{2 r}-1\right)\left(e^{r}+1\right)^{2}} d t \\
& =\sin k t_{0} M_{0}(k) .
\end{aligned}
$$

Again we see that $M_{0}(k)$ is a sine transform of an $L^{1}$ function:

$$
\begin{aligned}
\int_{-\infty}^{\infty}\left|\frac{\dot{r}(t) r^{2} e^{2 r}}{\left(e^{2 r}-1\right)\left(e^{r}+1\right)^{2}}\right| d t & =\int_{r(0)}^{r(\infty)} \frac{r^{2} e^{2 r}}{\left(e^{2 r}-1\right)\left(e^{r}+1\right)^{2}} d r \\
& =\int_{0}^{r(\infty)} \frac{r^{2} e^{2 r}}{\left(e^{2 r}-1\right)\left(e^{r}+1\right)^{2}} d r \\
& <\infty
\end{aligned}
$$

This is because $r^{2} e^{2 r} /\left(e^{2 r}-1\right)\left(e^{r}+1\right)^{2}$ is bounded on the interval $(0, r(\infty)]$. We see that $M_{0}(k)$ is again the sine transform of an odd $L^{1}$ function so that there exists an interval $k_{1} \leqq k \leqq k_{2}$ so that $M_{0}(k)$ is nonzero, giving us a Melnikov function with simple zeros. 
Under such a periodic stretching, we find chaotic dynamics occurring in the phase portrait of the evolution equations for the ellipse. This indicates a sort of randomness in the evolution of the vortex. The phase portrait includes a horseshoe as a subsystem that, as we know from $\S 2$, indicates somewhat erratic behavior on an invariant set. Assuming the inability to make completely precise measurements, we can only predict what will happen to the vortex for a finite time; after this time we have no knowledge of how it will evolve.

Appendix A. We present the details of the calculation of the following integral from $\S 3$ via residues:

$$
M_{0}(K)=\int_{-\infty}^{\infty} \frac{e^{\gamma t}-e^{-\gamma t}}{\left(e^{\gamma t}+\beta+e^{-\gamma t}\right)^{2}} \sin k t d t,
$$

which by a change of variables $\tau=\gamma t$ becomes

$$
M_{0}^{\prime}(m)=\frac{1}{\gamma} \int_{-\infty}^{\infty} \frac{e^{\tau}-e^{-\tau}}{\left(e^{\tau}+\beta+e^{-\tau}\right)^{2}} \sin m \tau d \tau,
$$

where $m=k / \gamma$. Consider the meromorphic function

$$
\frac{\left(e^{3 z}-e^{z}\right) e^{i m z}}{\left(e^{2 z}-\beta e^{z}+1\right)^{2}}
$$

The denominator has roots

$$
e^{z}=\frac{\beta \pm \sqrt{\beta^{2}-4}}{2}
$$

which we can write as

$$
=e^{ \pm i \alpha}
$$

since we know that $0<\beta<2$. Here, $\alpha=\cos ^{-1}(\beta / 2)$, which gives us $0<\alpha<\pi / 2$. Thus, the function

$$
\frac{\left(e^{3 z}-e^{z}\right) e^{i m z}}{\left(e^{z}-e^{i \alpha}\right)^{2}\left(e^{z}-e^{-i \alpha}\right)^{2}}
$$

has double poles at $z= \pm i \alpha+2 \pi i N, N \in \mathbb{Z}$. Let $r=z-(i \alpha+2 \pi i N)$. The integral is clearly odd in $m$. Thus we need only consider the case $m>0$. We have that

$$
\begin{aligned}
& \operatorname{Im} \frac{1}{\gamma} \int_{-\infty}^{\infty} \frac{e^{3 \tau}-e^{\tau}}{\left(e^{2 \tau}+\beta e^{\tau}+1\right)^{2}} e^{i m \tau} d \tau \\
& \quad=\lim _{N \rightarrow \infty} \operatorname{Im} \frac{1}{\gamma} \int_{-(2 N+1) \pi}^{(2 N+1) \pi} \frac{e^{3 \tau}-e^{\tau}}{\left(e^{2 \tau}+\beta e^{\tau}+1\right)^{2}} e^{i m \tau} d \tau \\
& \quad=\lim _{N \rightarrow \infty} \operatorname{Im} \frac{1}{\gamma}\left[\frac{1}{2 \pi i} \sum_{0<y<(2 N+1) \pi} \operatorname{Res}\left(\frac{e^{3 z}-e^{z}}{\left(e^{2 z}+\beta e^{z}+1\right)^{2}} e^{i m z}\right)\right. \\
& \left.\quad-\int_{|z|=(2 N+1) \pi, y>0} \frac{e^{3 \tau}-e^{\tau}}{\left(e^{2 \tau}+\beta e^{\tau}+1\right)^{2}} e^{i m \tau} d \tau\right]
\end{aligned}
$$

The last integral goes to zero as $N \rightarrow \infty$ so that for $m>0$, we wish to calculate

$$
\operatorname{Im}\left[\frac{2 \pi i}{\gamma} \sum_{y>0} \operatorname{Res}\left(\frac{e^{3 z}-e^{z}}{\left(e^{2 z}+\beta e^{z}+1\right)^{2}} e^{i m z}\right)\right] .
$$


Thus we need to calculate the residues of the function in the upper half-plane. We can calculate the coefficients of the Laurent expansion of the function by first considering the expansion of its components in the neighborhood of $i \alpha+2 \pi i N$. Writing $r=z-(i \alpha+2 \pi i N)$, we have

$$
\begin{gathered}
e^{3 z}=e^{3 i \alpha}\left(1+3 r+\frac{9}{2} r^{2}+\cdots\right), \\
e^{z}=e^{i \alpha}\left(1+r+\frac{1}{2} r^{2}+\cdots\right), \\
e^{i m z}=e^{-m \alpha-2 \pi m N}\left(1+i m r-\frac{m^{2}}{2} r^{2}+\cdots\right), \\
\left(e^{z}-e^{i \alpha}\right)^{2}=e^{2 i \alpha}\left(r^{2}+r^{3}+\cdots\right), \\
\left(e^{z}-e^{-i \alpha}\right)^{2}=-4 \sin ^{2} \alpha+4 i \sin \alpha e^{i \alpha} r+\cdots
\end{gathered}
$$

We write the function in the form

$$
\frac{1}{r^{2}}\left(\frac{a+b r+\cdots}{c+d r+\cdots}\right)
$$

which has the Laurent expansion

$$
\frac{a}{c} r^{-2}+\left(\frac{b}{c}-\frac{d a}{c^{2}}\right) r^{-1}+\cdots,
$$

so that the residue at $r=0$ is $b / c-d a / c^{2}$. Here

$$
\begin{aligned}
& a=\left(e^{3 i \alpha}-e^{i \alpha}\right) e^{-m \alpha-2 \pi m N}, \\
& b=e^{-m \alpha-2 \pi m N}\left[i m\left(e^{3 i \alpha}-e^{i \alpha}\right)+3 e^{3 i \alpha}-e^{i \alpha}\right], \\
& c=e^{2 i \alpha}\left(-4 \sin ^{2} \alpha\right), \\
& d=e^{2 i \alpha}\left(4 i e^{i \alpha} \sin \alpha-4 \sin ^{2} \alpha\right) .
\end{aligned}
$$

Let $R_{x}$ denote the residue at the point $x$. Then,

$$
R_{i \alpha+2 \pi i N}=\frac{m e^{-m \alpha-2 \pi m N}}{2 \sin \alpha} .
$$

Notice that $R_{i \alpha+2 \pi i N}=R_{i \alpha} e^{-2 \pi N m}$. A similar calculation shows that

$$
R_{-i \alpha+2 \pi i N}=\frac{-m e^{m \alpha-2 \pi m N}}{2 \sin \alpha} .
$$

We add the residues in the upper half plane to obtain $M_{0}^{\prime}(m)$ for $m>0$, and exploit the fact that $M_{0}^{\prime}(m)$ is odd in $m$ to obtain $M_{0}^{\prime}(m)$ for $m<0$. Thus for $m \neq 0$, our integral becomes

$$
M_{0}^{\prime}(m)=\frac{2 \pi}{\gamma}\left[\frac{m e^{-|m| \alpha}}{2 \sin \alpha}-\frac{m \sinh |m| \alpha}{\sin \alpha} \frac{e^{-2 \pi|m|}}{1-e^{-2 \pi|m|}}\right] .
$$

Acknowledgments. I would like to credit Prof. Andrew Majda of Princeton University for originally suggesting the idea of extending the Melnikov theory to study the onset of chaos in fluid flows with heteroclinic orbits. I would also like to thank him for the guidance he has given me on my A.B. thesis, from which this paper stems. I would like to thank AT\&T Bell Laboratories at Murray Hill, New Jersey, where I spent the summers of 1987 and 1988, for supplying me with the diagrams. 


\section{REFERENCES}

[1] L. V. Ahlfors, Complex Analysis, McGraw-Hill, New York, 1979.

[2] V. I. ARnold, Mathematical Methods of Classical Mechanics, Springer-Verlag, New York, 1984.

[3] V. I. Arnold And A. Avez, Ergodic Problems of Classical Mechanics, W. A. Benjamin, New York, Amsterdam, 1968.

[4] A. L. BertozzI, An extension of the Smale-Birkhoff homoclinic theorem, Melnikov's method, and chaotic dynamics in incompressible fluids, A.B. thesis, Princeton University, Princeton, NJ, 1987.

[5] G. D. Birk hoff, Nouvelles recherches sur les systèmes dynamiques, Mem. Pont Acad. Sci. Novi Lyncaei, 1 (1935), pp. 85-216.

[6] A. J. Chorin And J. E. Marsden, A Mathematical Introduction to Fluid Mechanics, Springer-Verlag, New York, 1979.

[7] J. Guckenheimer, A Brief Introduction to Dynamical Systems, Lectures in Applied Mathematics 17, Springer-Verlag, Berlin, New York, 1979, pp. 187-252.

[8] J. Guckenheimer And P. Holmes, Nonlinear Oscillations, Dynamical Systems, and Bifurcations of Vector Fields, Springer-Verlag, New York, 1983.

[9] M. W. Hirsch And S. Smale, Differential Equations, Dynamical Systems, and Linear Algebra, Academic Press, New York, 1974.

[10] P. J. Holmes, Averaging and chaotic motions in forced oscillations, SIAM J. Appl. Math., 38 (1980), pp. 68-80.

[11] - "Space and Time Periodic Perturbations of the sine-Gordon Equation, in Dynamical Systems and Turbulence, D. A. Rand and L.-S. Young, eds., Lecture Notes in Mathematics 89, SpringerVerlag, New York, Berlin, 1981.

[12] Holm, MARSDen, ANd Ratiu, Nonlinear stability of the Kelvin-Stuart cat's eyes flow, in AMS Proc. Math. Bio. Symposia and Summer Seminars, July, 1979.

[13] Y. Katznelson, An Introduction to Harmonic Analysis, Dover, New York, 1976.

[14] S. KIDA, J. Phys. Soc. Japan, 50 (1981), pp. 3517-3520.

[15] H. LAMB, Hydrodynamics, Dover, New York, 1945.

[16] A. MAJDA, Lectures on Incompressible Fluid Flow-Fall 1986, Princeton, NJ, 1986.

[17] J. E. MARSDEN, Chaotic orbits by Melnikov's method: a survey of applications, PAM-173/COMM, Center for Pure and Applied Mathematics, University of California, Berkeley, CA, August 1983.

[18] V. K. Melnikov, On the stability of the center for time periodic perturbations, Trans. Moscow Math. Soc., 12 (1963), pp. 1-57.

[19] J. Moser, Stable and Random Motions in Dynamical Systems, Princeton University Press, Princeton, NJ, 1973.

[20] D. W. MoOre AND P. G. SAFFMAN, The density of organized vortices in a turbulent mixing layer, J. Fluid Mech., 69 (1975), pp. 465-473.

[21] J. C. NEU, The Dynamics of a Columnar Vortex in an Imposed Strain, MRSI 022-83, 1983.

[22] - The dynamics of stretched vortices, J. Fluid Mech., 143 (1984), pp. 253-276.

[23] J. Palis, On Morse-Smale dynamical systems, Topology, 8 (1969), pp. 385-405.

[24] J. Pedlosky, Geophysical Fluid Dynamics, Springer-Verlag, New York, 1982.

[25] A. P. Prudnikov, Y. A. Brychkov, And O. I. Marichev, Integrals and Series: Vol. I. Elementary Functions (translated from Russian by N. M. Queen) Gordon and Breach, New York, 1986.

[26] S. SMALE, Differomorphisms with many periodic points, in Differential and Combinatorial Topology, S. S. Cairns, ed., Princeton University Press, Princeton, NJ, 1963, pp. 63-80.

[27] J. T. StuArt, Stability Problems in Fluids, AMS Lectures in Applied Mathematics 13, American Mathematical Society, Providence, RI, 1971, pp. 139-155. 